\title{
Fuzzy logic estimation of mosquito populations in homes in kibera slums, nairobi, kenya
}

\begin{abstract}
Pathogens transmitted by mosquitoes are a major cause of mortality and morbidity in human populations in developing countries. The most effective way of eliminating the risk of transmission of mosquito-borne diseases, such as malaria, is by reducing the number of mosquitoes. ProVector ${ }^{\circledR}$ mosquito control devices were placed in homes in the village of Kibera, Kenya. Villagers recorded their observations, biweekly, on the mosquito population during a 14 week period and significant differences $(\mathrm{P}<0.05)$ over time were found using parametric statistics. Lingual descriptors were assigned to fuzzy memberships, then defuzzified to numeric values based upon the number of mosquitoes collected from Ahero and Nyalenda, Kenya. A Sugeno-Takagi crisp controller model was developed and identified 11.1 weeks as the optimal point in time to replace the ProVector bait pad to prevent mosquito populations from recovering to severe levels predicted at week 13.4 (40 mosquitoes/ 5 minutes). This study provides a new method of estimating mosquito populations in areas where directly sampling mosquitoes may be difficult due to logistical or safety concerns.
\end{abstract}

Volume I Issue 3 - 2017

\author{
Thomas M Kollars,' Jason W Kollars, ${ }^{2}$ Mary \\ Kinoti,' Julius Kithinji \\ 'Department of Health Sciences, Liberty University, USA \\ ${ }^{2}$ Department of Computer Sciences, USA
}

Correspondence: Thomas M Kollars, Department of Health Sciences, Liberty University, USA, Email tkollars@liberty.edu

Received: September 20, 2017 | Published: November 02, 2017
Abbreviations: IVM, integrated vector management; BTI, bacillus thuringiensis israelensis

\section{Introduction}

Mosquito-borne diseases have killed more people than all wars combined. Various Integrated Vector Management (IVM) methods have been used to reduce the risk of these diseases by reducing the population of mosquitoes, e.g. insecticides, bed nets, repellents, traps. New technologies have been developed using attractant toxic sugar baits, such as the Provector Flower. ${ }^{1}$ The Pro vector ${ }^{\circledR}$ system uses an Entobac, a honey formulated with Bacillus thuringiensis israelensis (BTI) to attract and kill adult mosquitoes. The adult mosquitoes ingest the BTI and die within 3 to 7 days. If the adult mosquitoes die and land in larvae breeding sites, the larvae are also eliminated. ${ }^{2}$ Entobac is non-toxic and is the only pesticide which kills both adult and larvae mosquitoes. Conducting population studies of mosquitoes can be time consuming and expensive, particularly when conducting international studies. One system used to estimate mosquito numbers uses surveys of heads-of-house-holds using levels of annoyance. ${ }^{3}$ This study identifies a new method of estimating mosquito populations based upon lingual descriptors provided by the local citizens of the Kibera Slums, Nairobi, Kenya.

\section{Methods}

Anonymous residents of Kibera Slums, Nairobi, Kenya participated in the study $(\mathrm{N}=146)$. Residents were instructed on how to put together the proVector flowers and placement in homes. Each two weeks participants were asked to estimate the number of mosquitoes in their homes using the descriptors: very many, many, few, and very few and none. These descriptors are based on actual population numbers gathered from a separate study in Kenya. ${ }^{4}$ Personnel for the study was provided by the Riziki Foundation. Fuzzy logic using Statistica (Statsoft) was used to quantify and analyze lingual descriptors of mosquito populations in a community in order to test efficacy and length of control of the ProVector Applicator with Entobac pesticide. Lingual descriptors were assigned to fuzzy memberships, then defuzzified to numeric values based upon the number of mosquitoes collected from Ahero and Nyalenda, Kenya. Mosquitoes were collected at five minute intervals using backpack aspirators in 32 homes in Ahero and Nyalenda, Kenya in collaboration with the U.S. Army Kenya Medical Research Unit. The variation in numbers of mosquitoes collected in these two villages provided a basis for lingual descriptors for the fuzzy logic model. The Takagi Sugeno fuzzy logic has been used for various engineering systems but also in biological systems, e.g. identifying foraging strategies of bacterial species. ${ }^{5}$ A Sugeno-Takagi crisp controller model was developed and identified 11.1 weeks as the optimal point in time to replace the proVector bait pad to prevent mosquito populations from recovering to severe levels (40 mosquitoes/5 minutes) (Figure 1).

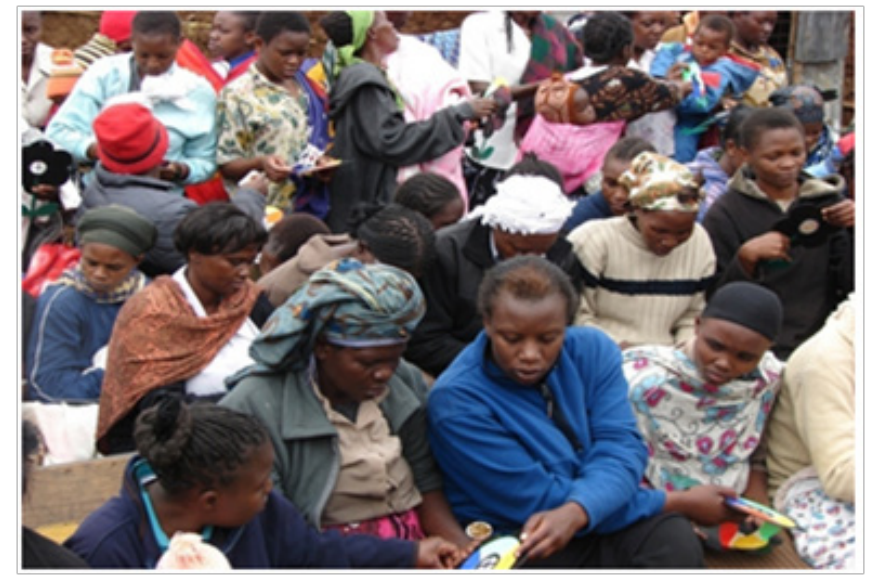

Figure I Mothers with infant children volunteered putting together ProVector Flowers for placement in their homes in the Kibera Slums, Nairobi, Kenya.

\section{Results}

Feedback from heads of households was high during the beginning of the study and was greatly reduced over the study and began to 
rebuild during the last few weeks. Mosquitoes began to decline in homes after two weeks and continued to be reduced until week 10 (Figure 2). After week 10 the mosquito population began to increase as bait was used up (Figure 3). Sugeno-Takagi crisp controller model was developed and predicted 11.1 weeks as the optimal point in time to replace the ProVector bait pad to prevent mosquito populations from recovering to severe levels, predicted at week 13.4 (very high $=40$ mosquitoes $/ 5$ minutes)

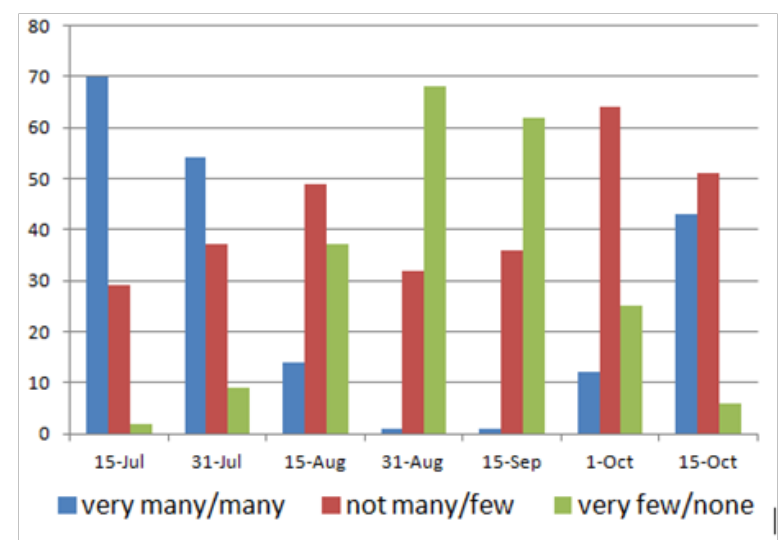

Figure 2 Percent of Homes Reporting Mosquito Abundance after Using ProVector Bait in Kibera, Kenya.

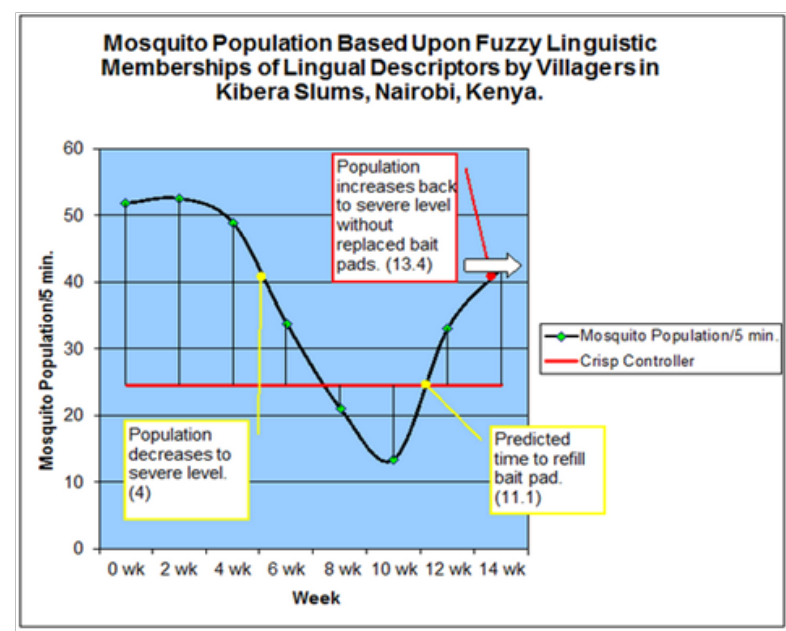

Figure 3 Decline of Mosquito Numbers after Placement of ProVector in Homes in Kibera, Nairobi, Kenya with 3 month Entobac bait pad (Survey Courtesy Riziki Foundation).Arrow depicts Takagi Sugeno crisp control model to replace bait pads to reduce predicted increase.

\section{Discussion}

The ProVector mosquito control device provides an eco-friendly and inexpensive means of controlling populations. Surveys of home owners in Kibera, Nairobi, Kenya were effective because of the willingness of home owners to participate, as they recognize the severity of mosquito-borne diseases. As an example, one mother asked the participating nurse to please thank the team, saying, "I can buy food for my children now instead of malaria medicine." Fuzzy logic and the Takagi Sugeno Model provided were effective in quantifying qualitative lingual descriptors to estimate mosquito populations and determine the effectiveness of the proVector technology. The ProVector Entobac bait pads cost about \$1.50 USD and identifying the optimal time for replacement could reduce cost of replacing too often or at the other end of the spectrum, not replacing frequently enough, possibly adversely affecting family health. In order to assist in supply logistics to remote regions, identifying the best time to replace the bait pads can optimize logistics. The Takagi Sugeno model has been used to optimize medical counter measures should a criminal or terrorist release of Shigella species occur. ${ }^{1}$ The Sugeno Takagi Crisp Controller can provide a useful tool in IVM planning and logistics by providing accurate estimates of when refills should be replaced or other forms of mosquito control should be implemented.

\section{Acknowledgements}

The authors would like to express gratitude first to the families that participated in this study and the Methodist Church of Kenya. The views expressed in this publication are those of the authors and do not reflect the official policy of the United States Government.

\section{Conflict of interest}

The author declares no conflict of interest.

\section{References}

1. Kollars TM, Hatfill S, Kollars EL, et al. Controlling mosquito populations and reducing the risk of mosquito-borne diseases in a beach resort of Punta Cana using eco-friendly technologies. J Pharma Biol. 2016;11(3):72-75.

2. Kollars TM. Application of Modeling to Optimize Medical Counter Measures to Reduce the Risk of Social Disruption from a Terrorist/ Criminal Attack in Restaurants in Kolkota, India. J Dent Med Sci. 2016;15:23-24.

3. Read NR, Rookert JR, Gathman JP. Public perception of mosquito annoyance measured by a survey and simultaneous mosquito sampling. JAMCA. 1994;10(1):79-87.

4. Yalwala S, Kollars JW, Kasembeli G, et al. Preliminary report on the reduction of adult mosquitoes in housing compounds in western Kenya, using Entobac biopesticide and the ProVector Flower. $J$ Med Entomol. 2016;83:1252-1244.

5. Mishra S. A hybrid least square-fuzzy bacterial foraging strategy for harmonic estimation. IEEE. 2005;9(1):61-73. 